\title{
Impaired Biomechanical Properties of Diabetic Skin
}

\author{
Implications in Pathogenesis of Diabetic Wound \\ Complications
}

\author{
Dustin M. Bermudez, ${ }^{\star \dagger}$ Benjamin J. Herdrich, * \\ Junwang Xu, ${ }^{\ddagger}$ Robert Lind, ${ }^{\S}$ David P. Beason, \\ Marc E. Mitchell, ${ }^{\ddagger}$ Louis J. Soslowsky, ${ }^{\text {ๆ }}$ and \\ Kenneth W. Liechty ${ }^{\ddagger *}$ \\ From the Department of Surgery," and the McKay Orthopedic \\ Research Laboratory, ${ }^{S}$ University of Pennsylvania School of \\ Medicine, Philadelphia, Pennsylvania; The Center for Fetal \\ Research, ${ }^{\dagger}$ The Children's Hospital of Philadelphia, Philadelphia, \\ Pennsylvania; the Department of Surgery, ${ }^{\ddagger}$ University of \\ Mississippi Medical Center, Jackson, Mississippi; and the \\ Department of Surgery, ${ }^{\S}$ University Medical Center of Groningen, \\ Groningen, The Netherlands
}

Diabetic skin is known to have deficient wound healing properties, but little is known of its intrinsic biomechanical properties. We hypothesize that diabetic skin possesses inferior biomechanical properties at baseline, rendering it more prone to injury. Skin from diabetic and nondiabetic mice and humans underwent biomechanical testing. Real-time PCR was performed for genes integral to collagen synthesis and degradation. MMP-2 and MMP-9, and TIMP-1 protein levels were assessed by ELISA and zymography. Collagen I and III content was assessed using Western blot analysis. At baseline, both murine and human diabetic skin was biomechanically inferior compared to nondiabetic skin, with decreased maximum stress and decreased modulus $(P<0.001$ and $<0.05$, respectively). Surprisingly, the expression of genes involved in collagen synthesis were significantly up-regulated, and genes involved in collagen degradation were significantly down-regulated in murine diabetic skin $(P<0.01)$. In addition, MMP-2 and MMP-9/TIMP-1 protein ratios were significantly lower in murine diabetic skin $(P<0.05)$. Collagen I levels and I:III ratios were lower in diabetic skin $(P<$ 0.05). These findings suggest that the predisposition of diabetics to wounds may be the result of impaired tissue integrity at baseline, and are due, in part, to a defect in the regulation of collagen protein synthesis at the post-transcriptional level. (Am J Pathol 2011, 178 : 2215-2223; DOI: 10.1016/j.ajpath.2011.01.015)
Diabetes is associated with increased morbidity and mortality, ${ }^{1}$ as well as impaired wound healing. ${ }^{2}$ Diabetesrelated admissions accounted for $22 \%$ of all hospital inpatient days in 2007, and diabetic foot ulcers account for $20 \%$ of all hospital admissions in diabetic patients, which are the leading cause of lower extremity amputations. ${ }^{2}$ Improvements made in diabetic wound management and prevention clearly have the potential to affect a large number of patients and decrease diabetic-related health care expenditures.

More than 100 factors have been identified that contribute to the impairment in diabetic wound healing. ${ }^{3}$ Decreased angiogenesis, ${ }^{4}$ impaired growth factor production, ${ }^{5}$ an altered inflammatory and immune response, ${ }^{5}$ a decreased rate of wound contraction, ${ }^{6}$ and an imbalance between the accumulation of extracellular components and their remodeling by matrix metalloproteinases (MMPs) $)^{7,8}$ have all been demonstrated in diabetic wounds. MMP-2 and MMP-9 have been shown to be present in greater concentration in wounded diabetic animals than their nondiabetic littermates, which is similar to findings from patients with nonhealing ulcers. ${ }^{9}$

Diabetes is characterized by significantly increased cross linking and nonenzymatic glycation of collagen, as well as elevated levels of advanced glycation end products (AGEs). ${ }^{10,11}$ Blockade of the receptor for advanced glycation end products (RAGEs) can restore the wound healing properties of diabetic (Db/Db) mice. ${ }^{12}$ Hyperglycemic animals have been shown to have significantly higher concentrations of glycated collagen and higher levels of collagenase activity. ${ }^{9,13}$ Furthermore, diabetic (Db/Db) mice have also been shown to have a prolonged inflammatory phase with sustained expression of the inflammatory cytokines macrophage chemoattractant protein 1 (MPC-1) and macrophage inflammatory protein 2 (MIC-2). ${ }^{14}$

\footnotetext{
The research presented in this article was supported in part by two grants from the National Institutes of Health: \# R56DK080672-01A1 (R56) and \# DP2DK083085-01 (diabetes pathfinder grant).

Accepted for publication January 25, 2011

Address reprint requests to Kenneth W. Liechty, M.D., 2500 N. State St., Guyton Building, University of Mississippi Medical Center, Jackson, MS 39216. E-mail: kliechty@UMC.edu.
} 


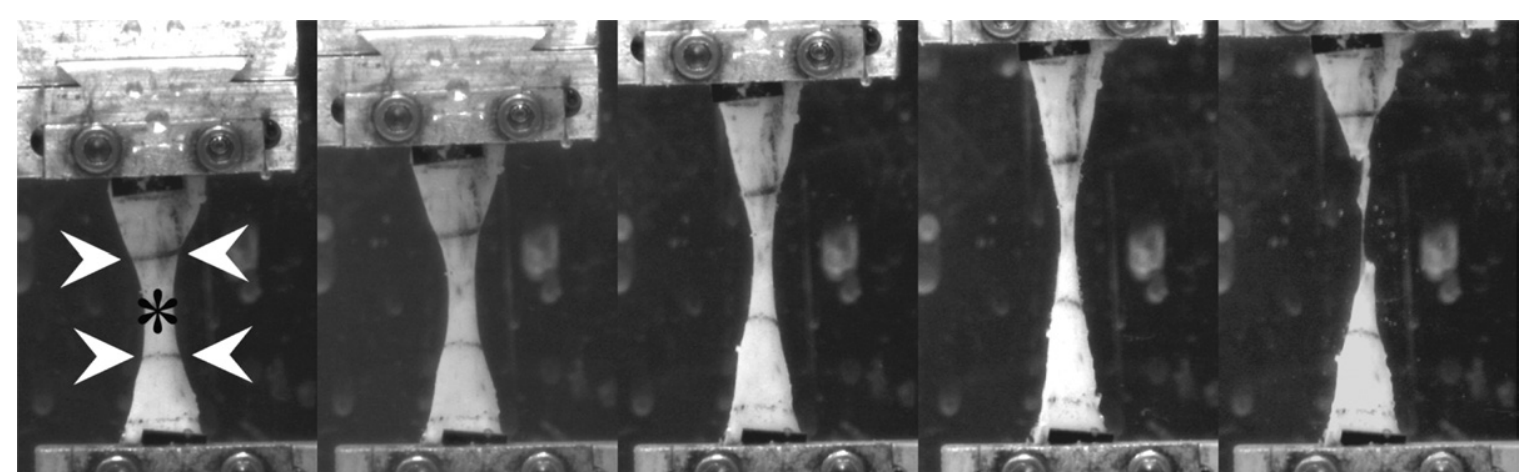

Figure 1. Five serial photographs demonstrating the biomechanical testing of skin samples. Arrowheads indicate the Verhoeff stain lines used to calculate the optical strain. An asterisk indicates the location of the scar when wounded skin was tested.

The biomechanical properties of diabetic skin are another critical aspect of wound-healing physiology. Diabetic, hyperglycemic rats were found to have inferior biomechanical properties than euglycemic rats after injury. ${ }^{15}$ Other studies confirm inferior properties after wounding, but few have assessed these properties at baseline. We hypothesize that there exist inherently inferior biomechanical properties of diabetic skin at baseline. Furthermore, we propose that these inferior biomechanical properties are the result of imbalances in collagen synthesis and degradation in diabetic compared to nondiabetic skin.

\section{Materials and Methods}

\section{Animals}

The animals were 6-week- to 8-week-old, genetically diabetic female C57BKS.Cg-m $+/+L e p r^{d b} / J$ mice $(\mathrm{db} / \mathrm{db})$ and age-matched, nondiabetic $(\mathrm{db} /+)$, heterozygous controls were obtained from the Jackson Laboratory (Bar Harbor, $\mathrm{ME})$. The mice were acclimated to the animal facility and subsequently wounded at 10 to 14 weeks of age. At the time of experiments, diabetic $(\mathrm{db} / \mathrm{db})$ mice weighed $>45 \mathrm{~g}$ with blood glucose in excess of $400 \mathrm{mg} / \mathrm{dL}$. db/+ mice weighed $<25 \mathrm{~g}$ with blood glucose $<250 \mathrm{mg} / \mathrm{dL}$. All animals received standard rodent chow and water ad libitum. All experimental protocols were approved by the Institutional Animal Care and Use Committee, and followed the guidelines described in the National Institutes of Health Guide for the Care and Use of Laboratory Animals.

\section{Murine Skin Harvest and Wounding Model}

Diabetic (Db/Db) and nondiabetic (Db/+) mice were euthanized with inhaled carbon dioxide. Unwounded dorsal skin was harvested and marked to preserve cranial-caudal orientation. A subset of diabetic (Db/Db) and nondiabetic $(\mathrm{Db} /+)$ mice were prepared for wounding. They were anesthetized with isoflurane and the dorsal skin was shaved and depilated. The skin was then cleaned with ethanol and then betadine. An 8-mm, full-thickness excisional dermal wound was performed through the panniculus carnosus using a dermal punch (Miltex, Inc., York, PA) on their dorsal skin at the same cranial-caudal level.
The wounds were covered with Tegaderm (3M, St. Paul, MN) and subsequently removed on postoperative day 2 . The mice were injected with the analgesic Banamine (Schering-Plough Animal Health Corp., Union, NJ) to control postoperative pain. Biomechanical testing took place 4 or 7 weeks after wounding. At harvest the dorsal skin was shaved and depilated and a full thickness skin area, including the wounded area, was excised and marked for cranial-caudal orientation.

\section{Murine Skin Biomechanical Testing}

Biomechanical testing was performed at baseline, 4 weeks, or 7 weeks after wounding on the murine diabetic and nondiabetic skin. After harvesting skin samples, they immediately underwent biomechanical testing. All samples had their subcutaneous tissue removed and a uniform dumbbell-shaped testing unit was stamped out using well-established techniques. ${ }^{16}$ Cranial-caudal orientation was preserved and the healed wound, if present, was centrally located within the testing unit. Figure 1 displays the device that we used to test each skin sample and then calculated modulus of elasticity, as well as maximum stress until failure.

Two Verhoeff stain lines were placed on either end of the dumbbell shape (Figure 1), demarcating the gauge length of the testing area. We measured the cross-sectional area of each sample first, by using a custom laserbased device that translates the tissue underneath a charged coupled device laser into a measurement of thickness. ${ }^{17}$ Position data using two linear variable differential transformers were used in conjunction with the thickness data to calculate an average cross-sectional area within the gauge length. Thus, the mean crosssectional area of the sample tested was calculated as precisely as possible to ensure that an accurate modulus and maximum stress were calculated.

The ends of the testing unit were fixed between sandpaper using a cyanoacrylate adhesive to prevent slipping. The testing unit was mounted in custom designed fixtures and tested in tension using an Instron 5543 test frame (Instron Corp., Norwood, MA). Each testing unit underwent a previously established protocol. ${ }^{18}$ In short, the testing unit was submerged in a $37^{\circ} \mathrm{C}$ PBS bath, 
preloaded to $0.005 \mathrm{~N}$, held for $120 \mathrm{~s}$, and then subjected to constant increase in force until the sample tears and can no longer hold tension. Using the stain lines, local tissue strain can be measured optically ${ }^{19}$ and analyzed using Matlab software (The Math Works, Inc., Natick, MA). Maximum stress to failure (a measure of tensile strength, calculated by dividing maximum load tolerated by the tissue sample divided by its cross-sectional area) was calculated using linear regression from the linear region of the stress-strain curve. The modulus of elasticity (another measure of tensile strength) was calculated from the optical strain that was calculated. Optical strain was calculated from the change in distance of the Verhoeff stained lines with the change in force applied to the tissue sample.

\section{ELISA Analysis of Murine Skin}

Skin samples from diabetic and nondiabetic mice $(n=5$ for each group) were morcellized and subsequently homogenized in a solution of $750 \mu \mathrm{L}$ of tissue lysis buffer (Qiagen, Inc., Valencia, CA). All subcutaneous tissue was removed prior to homogenization. This solution was then centrifuged at 10,000 rpm for 10 minutes. The supernatant was collected and frozen at $-80^{\circ} \mathrm{C}$ for further analysis. Protein concentration was quantified using a bicinchoninic acid protein assay (Thermo Scientific, Rockford, $\mathrm{IL}$ ) that uses a standard curve generated from known concentrations of bovine serum albumin (Thermo Scientific). All samples were run in duplicate. Enzyme-linked immunosorbent assay (ELISA) was then used to quantify the concentration of MMP-2, MMP-9, and tissue inhibitor metalloproteinase-1 (TIMP-1) protein levels (R\&D Systems, Minneapolis, MN). The ELISA kits that were used quantified the total amounts of MMP-2, MMP-9, and TIMP-1, regardless of whether it was bound to a TIMP or MMP or in its "pro" or active form. Values were normalized by the respective protein concentration of the samples.

\section{Extraction of Collagen and Western Blot for Collagens I and III}

Collagen was extracted using previously published methods ${ }^{20,21}$ with some modification. Briefly, skin samples were weighed (approximately $8 \mathrm{~mm}^{2}$ ) and cut into $1-\mathrm{mm}$ pieces and were subsequently homogenized in a $0.5 \mathrm{M}$ acetic acid solution containing $1 \times$ protease inhibitor cocktail and $5 \mathrm{mmol} / \mathrm{L}$ EDTA. This mixture effectively solubilizes total collagen by breaking up collagen cross links. Homogenates were then centrifuged for 4 hours at $4^{\circ} \mathrm{C}$ at $16.1 \times 10^{3} \mathrm{G}$. The lipid layer was aspirated and the process was repeated. The protein concentration of the supernatant was quantified with a bicinchoninic acid protein assay.

Western blot was performed with a standard technique. Final protein concentrations were standardized to $1 \mu \mathrm{g} / 2 \mu \mathrm{L}$ using a sample buffer and were subsequently boiled at $95^{\circ} \mathrm{C}$ for 5 minutes. Tris-acetate gels (3\% to 8\%) were run at $150 \mathrm{~V}$ for 1 hour and were then transferred at $30 \mathrm{~V}$ overnight at $4^{\circ} \mathrm{C}$. The membranes were rinsed with
Tris-buffered saline and were subsequently blocked with $5 \%$ milk in Tris-buffered saline with $0.1 \%$ Tween for 1 hour. Collagen I antibody (Abcam Inc., Cambridge, MA) was diluted to 1:2000 (collagen III to 1:1000) and blots incubated for 1 hour at room temperature and then washed with Tris-buffered saline with $0.1 \%$ Tween. They were subsequently incubated with secondary antibody (anti-rabbit IgG horseradish peroxidase; GE Healthcare, Piscataway, NJ) at 1:10,000 for 1 hour at room temperature. Western blots were washed with Tris-buffered saline with $0.1 \%$ Tween and then Tris-buffered saline. The blots were then developed using a standard chemiluminescence solution (ECL solution A and B; GE Healthcare) and were incubated for 1 minute. The film was initially exposed for 30 seconds and was adjusted accordingly.

\section{Real-Time PCR of Murine Diabetic and Nondiabetic Skin}

Total RNA was extracted and purified from skin samples of diabetic and nondiabetic mice ( $n=5$ for each group), previously obtained, after homogenization in TRIzol (Invitrogen, Life Technologies, Carlsbad, CA) and following the manufacturer's instructions. RNA was converted into cDNA using the SuperScript First-Strand Synthesis System (Invitrogen, Life Technologies). We used random primers for the reverse transcription reaction. Real-time quantitative PCR was performed with the ABI 7900 realtime PCR thermal cycler (Applied Biosystems, Foster City, CA). These samples were amplified in triplicate. Primers for col1a2, col3a1, col5a1, Hsp47, Smad3, Sp1, MMP-2, MMP-9, TIMP-1, and TIMP-2 were amplified according to manufacturer's instructions using the TaqMan gene expression assay (Applied Biosystems).

Internal normalization was achieved by using the housekeeping gene (18s) as an internal control. "Fold change" was calculated by comparing the mRNA expression for the $\mathrm{Db}$ mouse versus the heterozygote mouse for a particular gene. Each group was composed of five mice. Each experimental group's mean and SEM was calculated from these five biological replicates, not technical replicates. Results are reported from a single experiment averaged and reported as mean \pm SEM.

\section{Gelatin Zymography for MMP-2 and MMP-9 Activity}

Samples from unwounded skin were run for quantitative gelatin zymography to evaluate the proteolytic activity of the extracellular matrix (ECM). Homogenates of four diabetic samples and four heterozygote samples were run at room temperature using a Novex 10\% zymogram (gelatin) gel (Invitrogen, Life Technologies) using the manufacturer's instructions. Murine MMP-2 and MMP-9 standards (R\&D Systems) were loaded as controls for quantifying proteolytic activity. Whole protein was loaded to quantify activity of their respective proteins. We had used APMA during our initial experiments to demonstrate that we did indeed have some MMP activity (data not shown). We used whole protein to evaluate the ratios of 
pro-MMP to active MMP based on the size of the molecule relative to a molecular weight standard run at the same time. Protein concentration was normalized between samples by diluting with sample buffer. Gels were allowed to renature in the manufacturer's buffer for 1 hour and incubated overnight at $37^{\circ} \mathrm{C}$. Band intensity was quantified with densitometry and using a standard curve generated with increasing concentrations of the MMP-2 and MMP-9 standards.

\section{Acquisition of Human Skin, Biomechanical Testing}

All research was approved through the institutional review board at the Children's Hospital of Philadelphia. Human skin samples were collected postmortem from the anterior portion of the lower extremity of individuals with and without diabetes. The specimens were at least $5 \times 5 \mathrm{~cm}$ in size and were collected within eight hours of death through the National Disease Research Interchange. Samples were obtained from patients who were 55 to 75 years of age and did not have any known comorbid malignancy or a history of radiation or chemotherapy. Data such as the type of diabetes and current medications were included with each sample. Sun exposure (ie, a clear risk factor for skin with weaker tensile strength) was not known per se, although any history of melanoma would have been included in the patient summary. The skin samples were immediately flash frozen in liquid nitrogen. Once ready for analysis samples were brought to room temperature and all subcutaneous tissue was excised.

\section{Statistical Analysis}

Data are presented as mean \pm SEM to two significant figures. Statistical analysis was performed with oneway analysis of variance using SPSS 15.0 (SPSS, Inc., Chicago, IL). Values were transformed (In) when required to achieve normality of sampling. When significant differences $(P<0.05)$ were found, a least significant difference posthoc test, Mann-Whitney U-test, was used to identify differences between individual means.

\section{Results}

\section{Diabetic Skin Is Biomechanically Inferior}

At baseline, murine diabetic skin possesses significantly inferior biomechanical properties when compared to nondiabetic controls. The cross-sectional area for diabetic skin was greater than the heterozygote mice at baseline $\left(3.24 \pm 0.49 \mathrm{~mm}^{2}\right.$ versus $1.74 \pm 0.37 \mathrm{~mm}^{2}, P<0.001$; Figure 2A). We found that murine diabetic skin had a much lower maximum stress (Figure 2B). On average, the diabetic samples failed at $0.62 \pm 0.19 \mathrm{MPa}$ while the nondiabetic control samples failed at $2.10 \pm 0.49 \mathrm{MPa}$ $(P<0.001)$. Murine diabetic skin also possesses a decreased modulus of elasticity. On average, the modulus of the diabetic skin measured $1.82 \pm 1.18 \mathrm{MPa}$, compared to $7.72 \pm 3.00 \mathrm{MPa}$ for nondiabetic controls $(P<$ 0.001) (Figure $2 \mathrm{C}$ ).

\section{Diabetic Murine Dermal Scar Is Not Biomechanically Inferior}

At four weeks after wounding, healed murine diabetic wounds $(n=5)$ showed no significant difference in biomechanical properties when compared to nondiabetic wounds $(n=7)$. For the four-week postwounding samples, there was no significant difference between crosssectional area among the wounded diabetics $(2.46 \pm$ $0.46 \mathrm{MPa})$ and controls $(2.86 \pm 0.69 \mathrm{MPa} ; P=0.28$, Figure 2D). Diabetic maximum stress $(0.46 \pm 0.18 \mathrm{MPa})$ was similar to nondiabetic samples $(0.42 \pm 0.04 \mathrm{MPa}$; $P=0.56$; Figure $2 \mathrm{E})$. The modulus was also similar between diabetic $(1.49 \pm 0.29 \mathrm{MPa})$ and nondiabetic samples $(2.02 \pm 0.79 \mathrm{MPa} ; P=0.19$; Figure $2 \mathrm{~F})$.

Again at 7 weeks post-wounded, the healed diabetic excisional wounds $(n=9)$ showed similar biomechanical properties compared to nondiabetic control excisional wounds $(n=8)$. No significant difference between the diabetic and controls was found for cross-sectional area $(2.61 \pm 0.50 \mathrm{MPa}$ versus $2.75 \pm 0.60 \mathrm{MPa} ; P=0.61$; Figure 2D), maximum stress to failure $(0.58 \pm 0.23 \mathrm{MPa}$ versus $0.51 \pm 0.16 \mathrm{MPa} ; P=0.45$; Figure $2 \mathrm{E}$ ), or modulus $(3.31 \pm 1.41$ versus $3.78 \pm 2.10 \mathrm{MPa} ; P=0.59$; Figure 2F), respectively. Interestingly, the observation that diabetic skin restores its baseline biomechanical properties after injury, whereas nondiabetic skin does not, which is an intriguing finding. The significance of this finding, however, remains to be elucidated.

\section{Human Biomechanical Testing}

Similarly, human diabetic skin also demonstrated overall inferior biomechanical properties. Figure 2, G-I summarizes the results of the tensile strength testing of human diabetic $(n=4)$ and nondiabetic $(n=7)$ skin samples. There was no difference in mean cross-sectional area (Figure 2G). The average maximum stress for diabetic skin was significantly decreased compared to that of nondiabetic skin (4.14 MPa \pm 1.88 versus $6.52 \mathrm{MPa} \pm 1.71 ; P=0.03$, respectively; Figure $2 \mathrm{H}$. Additionally, the average modulus for diabetic skin samples was significantly lower than that for the nondiabetic skin samples $(12.8 \mathrm{MPa} \pm 5.28$ versus $25.2 \mathrm{MPa} \pm$ $11.8 ; P=0.04$; Figure 21).

\section{Expression of Genes Involved in ECM Degradation Are Decreased in Diabetic Skin}

Real-time PCR analysis of genes associated with extracellular matrix turnover demonstrated significant differences between diabetic and nondiabetic skin (Figure 3). MMP-9 gene expression was significantly decreased in diabetic mice compared to nondiabetic mice (5.21 \pm 2.07 versus $15.8 \pm 4.20$, respectively; $P<0.001$ ). TIMP-1 also demonstrated increased gene expression in diabetic mice $(9.09 \pm 2.71$-fold $)$ than in nondiabetic mice 
A

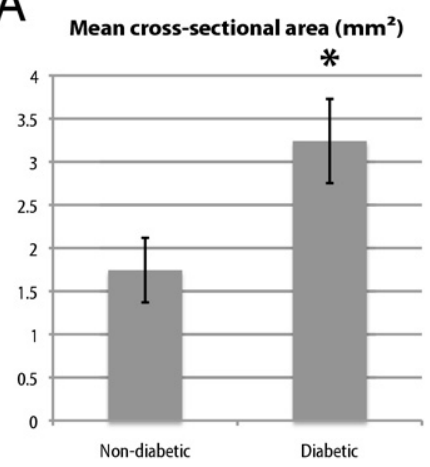

D

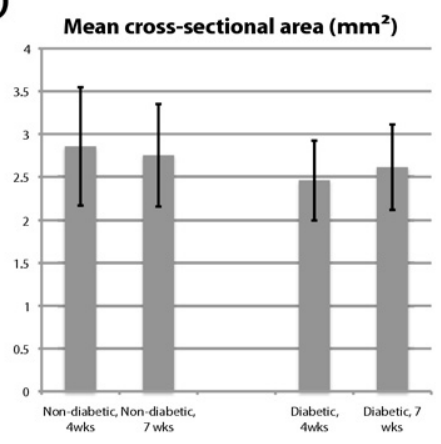

G

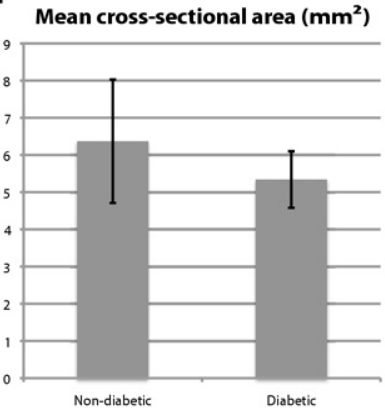

B

Mean maximum stress (MPa)

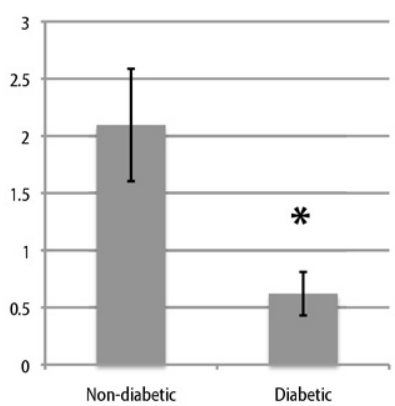

E

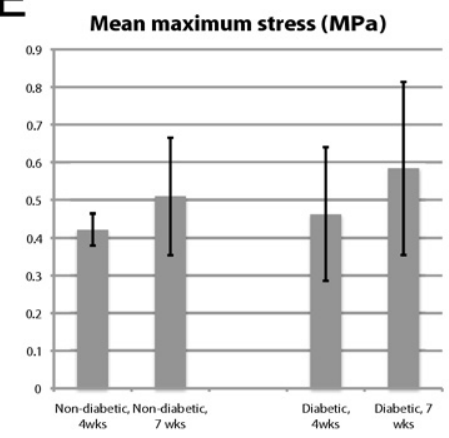

$\mathrm{H}$

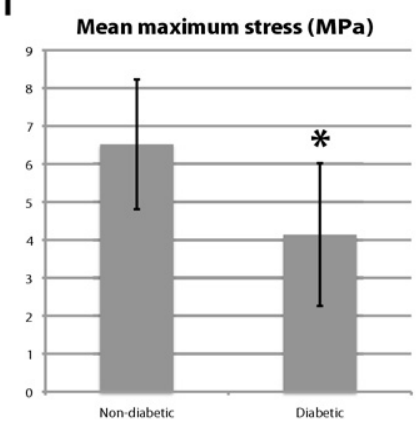

C

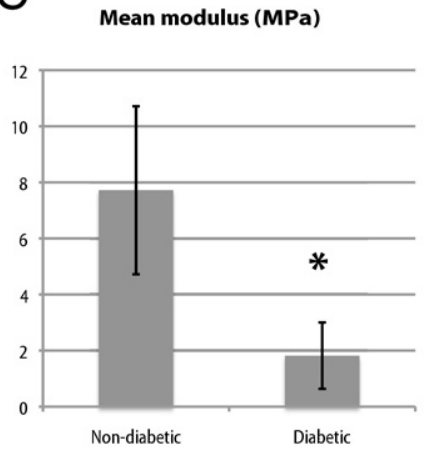

F

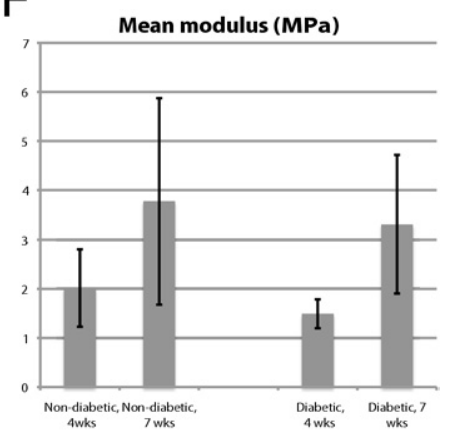

I

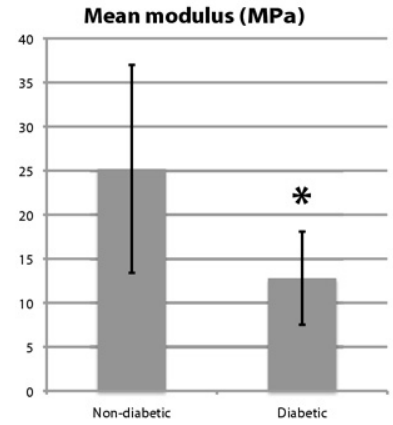

Figure 2. Biomechanical properties of diabetic and nondiabetic murine and human skin at baseline and at 4 and 7 weeks after wounding. A displays the mean cross-sectional area for murine diabetic and nondiabetic skin at baseline. B displays the maximum stress to failure, and $\mathbf{C}$ the modulus for diabetic mouse and nondiabetic mouse skin at baseline. At 4 weeks, and 7 weeks after wounding similar tensiometer testing was done, the mean cross-sectional area, maximal stress, and modulus are displayed for this group in $\mathbf{D}, \mathbf{E}$, and $\mathbf{F}$, respectively. Cross-sectional area, maximal stress, and modulus area also displayed for human skin at baseline in $\mathbf{G}, \mathbf{H}$, and $\mathbf{I}$, respectively). ${ }^{*} P<0.001$ when diabetic is compared to nondiabetic unwounded skin.

(2.31 \pm 0.522; $P<0.001)$. However, there was a mild increase in MMP-2 gene expression in the diabetic mouse skin (6.82 \pm 1.70 -fold) when compared with nondiabetic mouse skin, $(4.10 \pm 1.02 ; P=0.025)$. There was no significant difference in TIMP-2 gene expression between diabetic or nondiabetic mice (3.55 \pm 0.81-fold versus $2.85 \pm 0.65$, respectively).

\section{Factors Promoting ECM Degradation Are Decreased in Diabetic Skin}

ELISA analysis of the concentrations of proteins involved in the degradation of the extracellular matrix also revealed differences between diabetic and nondiabetic murine skin (Figure 4). Specifically, diabetic mouse skin had significantly decreased MMP-9 protein concentra- tions $(0.010 \pm 0.0024 \mathrm{ng} / \mu \mathrm{g})$ compared to nondiabetic mouse skin ( $0.019 \pm 0.0023 \mathrm{ng} / \mu \mathrm{g} ; P=0.0003$; Figure $4 \mathrm{~A})$. There was no significant differences in MMP-2 protein concentrations between diabetic and nondiabetic skin $(0.044 \pm 0.0071 \mathrm{ng} / \mu \mathrm{g}$ versus $0.041 \pm 0.016 \mathrm{ng} / \mu \mathrm{g}$, respectively; Figure $4 \mathrm{~B}$ ). In addition, TIMP-1 protein concentrations were also significantly increased in the diabetic mouse skin $(0.53 \pm 0.35 \mathrm{ng} / \mu \mathrm{g})$ compared to the nondiabetic mouse skin $(0.33 \pm 0.17 \mathrm{ng} / \mu \mathrm{g} ; P=0.04$; Figure $4 \mathrm{C})$. The ratio of MMP-9:TIMP-1 concentrations was also significantly different (Figure 4D).

ELISA analysis of MMP and TIMP proteins in human skin revealed differences similar to that found in the mouse ELISA. Diabetic human skin had significantly decreased MMP-9 levels compared to nondiabetic skin (0.00048 \pm $9.8 \times 10^{-4} \mathrm{ng} / \mu \mathrm{g}$ versus $0.0014 \pm 0.0017 \mathrm{ng} / \mu \mathrm{g}$, respec- 


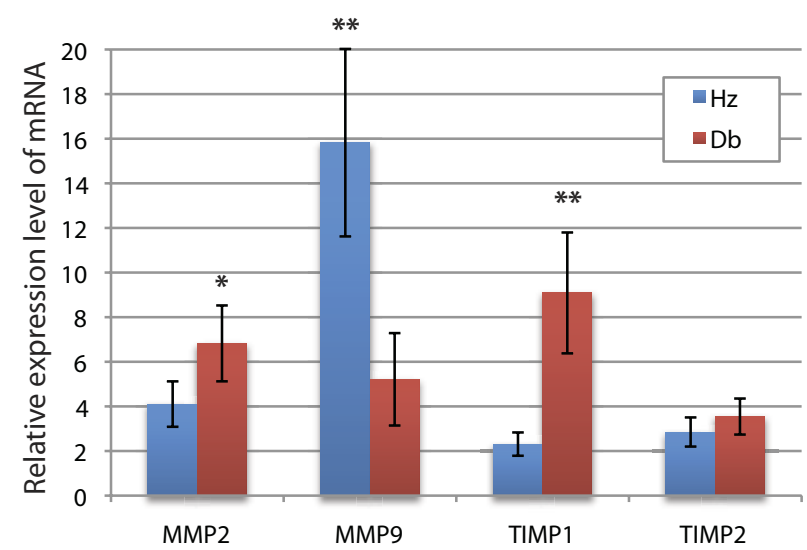

Figure 3. Real-time PCR of genes integral to the turnover of collagen 1, 3, and 5 in baseline murine diabetic and nondiabetic skin. Mean matrix metalloproteinases (MMP)9 was significantly greater among heterozygous mice when compared with diabetic mice but MMP2 and tissue inhibitor metalloproteinase (TIMP) 1 were greater among diabetic mice. There was no significant difference in the gene expression of TIMP2. $\mathrm{Db}=$ diabetic; $\mathrm{Hz}=$ heterozygous. ${ }^{*} P<0.05$. ${ }_{\text {*a* }} P<0.01$ when compared to lower value of the other group.

tively; $P=0.04$; Figure $5 \mathrm{~A}$ ). There was a greater ratio of MMP-9:TIMP-1 concentration in the human diabetic patients (Figure 5B). No significant difference in MMP-2 or TIMP-1 concentration between human diabetic and nondiabetic skin was observed (Figures 5, C and D, respectively).

\section{ECM Proteolytic Activity Is Decreased in Diabetic Skin}

Quantitative zymography demonstrated a significant difference in activity between MMP-2 and MMP-9 in

\section{A}

Mean [MMP9/protein]
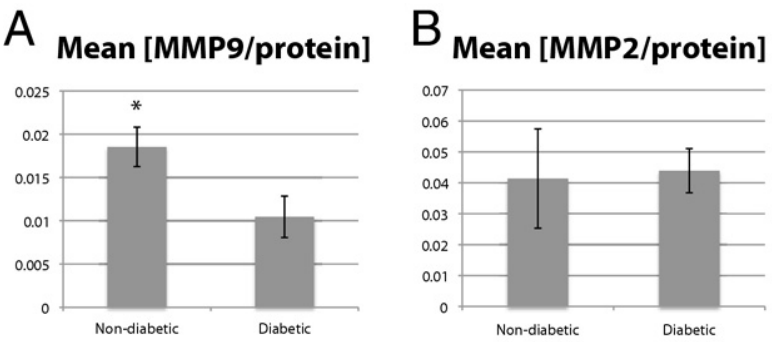

\section{C}

Mean [TIMP1/protein]
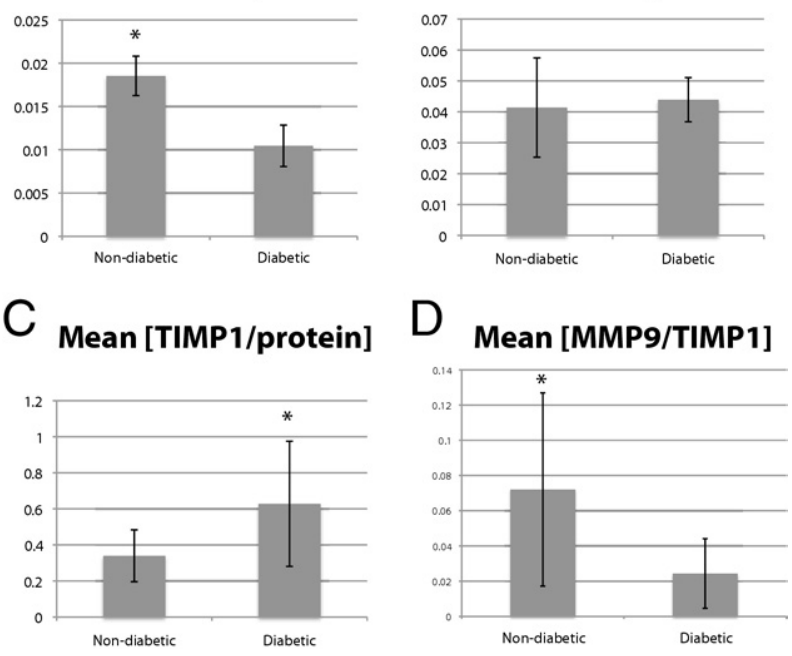

Figure 4. Extracellular matrix protein concentrations of unwounded diabetic and nondiabetic murine skin. The mean matrix metalloproteinases (MMP)9 concentration divided by overall protein concentration is displayed in $\mathbf{A} \quad\left({ }^{*} p=0.0003\right)$. Mean MMP2 and tissue inhibitor metalloproteinase (TIMP) 1 concentrations divided by overall protein concentrations are displayed in $\mathbf{B}$ and $\mathbf{C}$, respectively ( $\left.{ }^{*} p=0.04\right)$. The MMP9:TIMP1 ratio, an indicator of overall proteolytic activity, is displayed in $\mathbf{D}$.
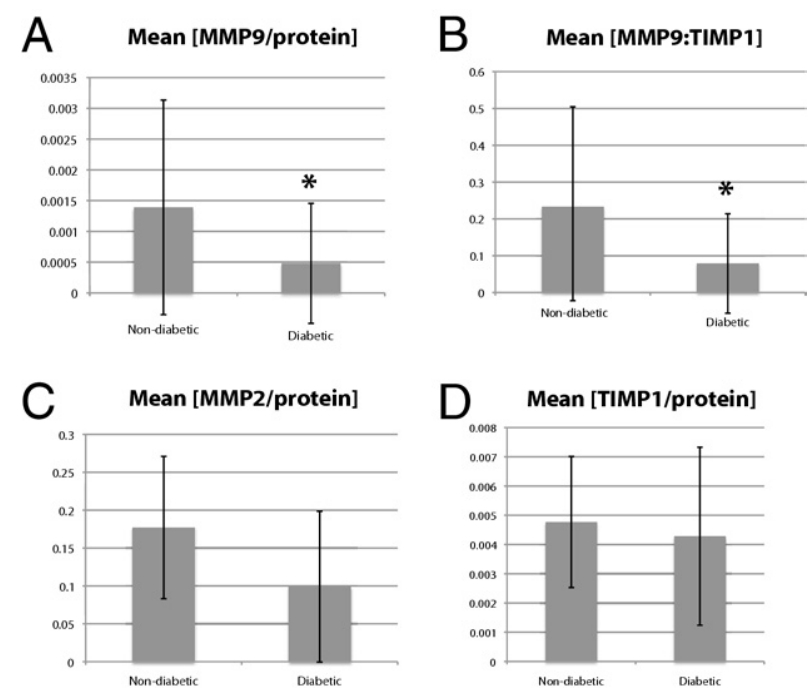

Figure 5. Extracellular matrix protein concentrations of unwounded diabetic and nondiabetic human skin. The mean matrix metalloproteinases (MMP)9 concentration divided by overall protein concentration is displayed in A. The MMP9:tissue inhibitor metalloproteinase (TIMP) 1 ratio, an indicator of overall proteolytic activity, is demonstrated in $\mathbf{B}$. Mean MMP2 and TIMP1 concentrations divided by overall protein concentrations are displayed in $\mathbf{C}$ and $\mathbf{D}$, respectively. ${ }^{*} P<0.04$ when diabetic is compared to nondiabetic unwounded skin.

nonwounded diabetic and heterozygote skin. Figure 6 (top portion) displays the zymogram with diabetic $(n=$ $4)$ and nondiabetic $(n=4)$ specimens. MMP-2:proMMP-2 ratios in the diabetic group were higher $(1.01 \pm$ $0.16)$ than the nondiabetic group $[0.44 \pm 0.23 ; P=$ 0.008 ; Figure 6 (bottom left)]. Active MMP-2 concentrations were significantly higher in the diabetic group as well [163 $\pm 58.8 \mu \mathrm{g} / \mathrm{mL}$ versus $78.6 \pm 42.9 \mu \mathrm{g} / \mathrm{mL}$, respectively; $P=0.025$; Figure 6 (bottom center)]. No active MMP-9 was found in the diabetic mice, whereas the heterozygotes had an average of $273 \mu \mathrm{g} / \mathrm{mL}$ [Figure 6 (bottom right)].

\section{Expression of Genes Involved in Collagen Synthesis Is Increased in Diabetic Skin}

Real-time PCR analysis of genes involved in collagen synthesis demonstrated significant differences between diabetic and nondiabetic skin. Specifically, the expression of collagens 1a2, 3a1, 5a1, and 5a2 were all significantly increased in diabetic mice in comparison to nondiabetic mice (Figure 7). Collagen 1a2 had $12.2 \pm 3.42-$ fold greater expression in diabetic mice when compared with nondiabetic mice $(4.38 \pm 1.42$-fold expression; $P=$ $0.001)$. Gene expression was also elevated in diabetic mice in the following genes: col3a1 (9.35 \pm 2.56 -fold versus $3.16 \pm 0.848 ; P<0.001)$, col5a1 ( $8.28 \pm 2.26$-fold versus $3.66 \pm 0.870 ; P=0.0027)$ and col5a2 (10.8 \pm 2.91 -fold versus $2.33 \pm 0.500 ; P<0.001$ ). Genes associated with collagen processing and chaperoning (Hsp47, Smad3, Sp1), however, showed no significant differences between the two groups. 


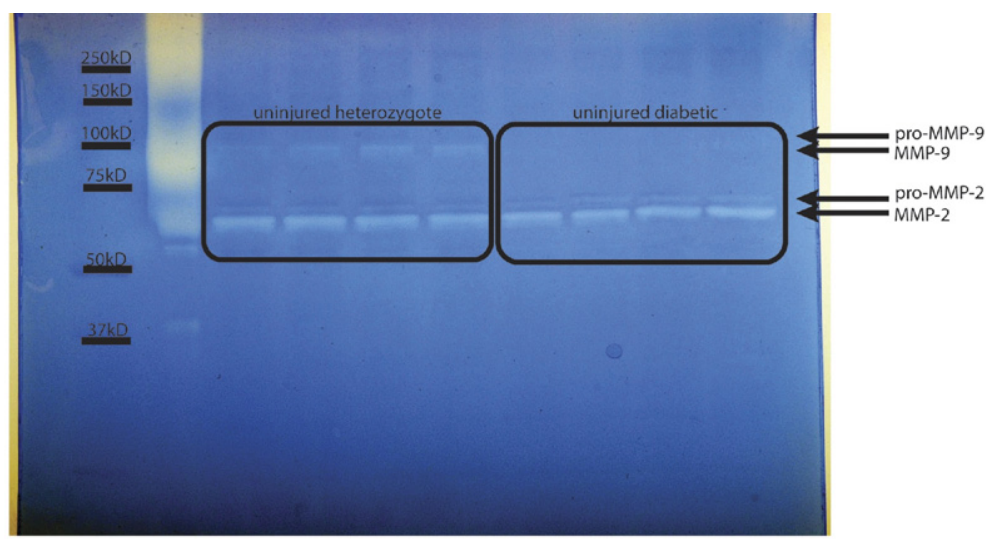

MMP-2:pro MMP-2

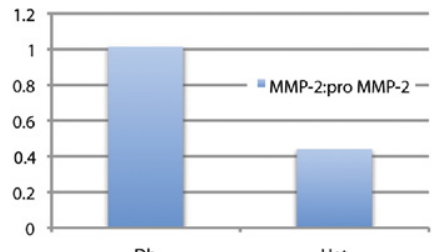

$\mathrm{Db}$
[MMP-2] ug/mL

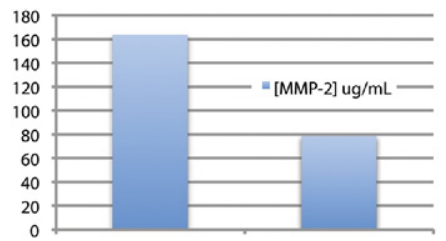

$\mathrm{Db}$

Het
[MMP-9] ug/mL

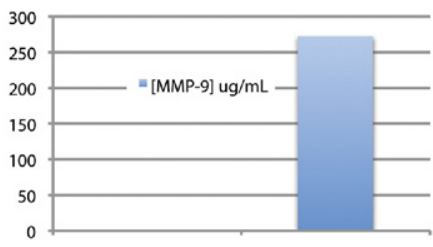

$\mathrm{Db}$

Het

Figure 6. Quanitative zymography for extracellular matrix (ECM) proteolytic activity. Top panel displays the zymogram ran with four samples of Het mice and four samples of $\mathrm{Db}$ mice. Each sample of protein homogenized dermis from unwounded animals. The bottom three graphs represent: the ratio of active mean matrix metalloproteinases (MMP)-2:pro-MMP-2, the total concentration of active MMP-2 and the total concentration of active MMP-9. Db, diabetic; Het, heterozygote nondiabetic.

\section{Decreased Collagens I and III Content in Diabetic Skin}

Western blot analysis of collagen types I and III demonstrated significant differences between diabetic and nondiabetic skin (Figure 8A). Murine diabetic skin $(n=4)$ had decreased type I collagen content compared to nondiabetic murine skin $(n=4),(0.12 \pm 0.064 \mu \mathrm{g}$ versus $0.19 \pm 0.082$, respectively; $P=0.11$ ). Diabetic murine skin also demonstrated increased type III collagen content compared to nondiabetic murine skin (0.010 \pm $0.0019 \mu \mathrm{g}$ versus $0.015 \pm 0.0013 \mu \mathrm{g}$, respectively; $P=$ 0.0081 ). It has been shown that the collagen I:III ratio can prognosticate the tensile strength of the ensuing wound or tissue. ${ }^{22,23}$ The absolute ratio of nondiabetic murine skin had a much greater collagen I:III ratio $(17 \pm 6.0)$ than diabetic murine skin $(8.0 \pm 3.6 ; P=0.018$; Figure $8 \mathrm{~B})$.

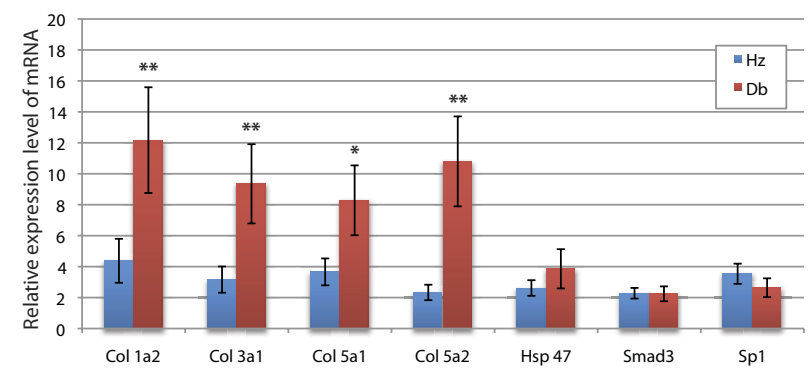

Figure 7. Real-time PCR of genes integral to the synthesis of collagens 1, 3, and 5 in baseline murine diabetic and nondiabetic skin. Once mRNA was amplified with real-time PCR, collagens 1a2, 3a1, 5a1, and 5a2 were all found to be significantly higher in diabetic skin $\left(\mathrm{n}=5{ }^{* * *} P<0.01\right.$ for all except col5a1, which was ${ }^{*} P<0.05$. Hsp 47, Smad3, and Sp1 had no significant differences. Db, diabetic; Hz, heterozygous.

\section{Discussion}

Our results indicate that diabetic skin (both murine and human cadaveric) is biomechanically inferior to nondiabetic skin at baseline. With wounding, however, there did not appear to be a significant difference in tensile strength after 4 or 7 weeks of healing. In an effort to further characterize the mechanisms involved with the diabetics' poorer tensile strength, we analyzed an array of factors involved in generating and degrading the extracellular matrix. Surprisingly, although the actual content of collagens I and III were lower in diabetics, the amount of transcription was far greater. We feel that this mechanistic analysis of the pathway of diabetic and normal wound healing demonstrates that the de-

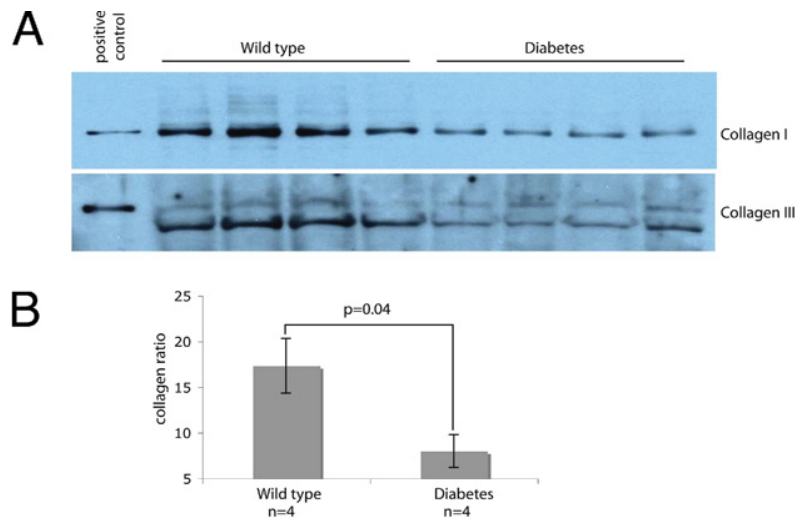

Figure 8. A: Western blot for collagen I and collagen III in diabetic and nondiabetic mice. There was a higher amount of collagen I in wild-type skin when compared to diabetic skin, whereas the reverse is true with respect to collagen III. B: When the collagen I:III ratio was calculated, the ratio was significantly higher in wild-type mice than in diabetic mice ( $17 \pm 6.0$ versus $8.0 \pm 3.6 ; P=0.04)$ 
creased tensile strength is likely due to disarrayed and decreased collagen deposition that undergoes post-transcriptional modification.

The majority of studies that have investigated the biomechanics of diabetic skin have examined it in the wounded state, not at the baseline. Two previous studies have addressed baseline biomechanical properties in diabetic human skin demonstrating greater stiffness and less extensibility. ${ }^{24,25}$ We show here that at baseline there are inherent differences in the biomechanical properties of the two tissues and that decreased tensile strength, collagen deposition, and post-transcriptional modification contribute to this difference in biomechanical properties. Decreased modulus, tensile strength, and collagen deposition all may contribute to predisposition to injury and poor wound healing found in diabetic skin.

The main component of the ECM responsible for structural integrity is type I collagen. Collagen content is regulated by synthesis of new collagen and degradation of existing collagen via MMPs. These enzymes are regulated by their own gene expression as well as by the production of TIMPs and inflammatory cytokines, such as tumor necrosis factor- $\alpha$. Our results demonstrate that there is decreased collagen content of the diabetic dermis. However, this decreased collagen content is associated with increased gene expression of enzymes involved in collagen synthesis and decreased production of factors that promote collagen degradation. These results suggest that the defect in collagen protein content in diabetic skin at baseline is at the post-transcriptional level.

Diabetics have a known wound healing impairment. Our biomechanical testing demonstrates that following injury, murine diabetic wounds have similar biomechanical properties to that of nondiabetic wounds. Although a direct comparison cannot be made to clinical data, these results suggest the defect in tissue integrity may be inherent to diabetic skin at baseline. The incidence of foot ulceration is $25 \%$ in diabetics, which is significantly higher than nondiabetics, suggesting that at baseline there is a predisposition to wounding, not justc delayed wound healing after trauma. ${ }^{26}$ Thus, factors that predispose the diabetic skin to injury may have an underappreciated role in the pathogenesis of the diabetic wound healing impairment.

Diabetic animal studies have demonstrated poorer biomechanical properties after wounding, as well as increased MMP-9, decreased TIMP-1, and a more proteolytic ECM. ${ }^{9,27,28}$ In vitro collagen matrices that were artificially glycated displayed a decrease in biomechanical properties when compared to nonglycated matrices both in vitro and when implanted in vivo. ${ }^{29}$ There has also been a suggestion that collagen breakdown may be altered in the setting of diabetes due to glycation and cross linkage. ${ }^{30}$ Impaired contraction in glycated collagen matrices is associated with decreased MMP activity. ${ }^{31}$

The diabetic wound healing impairment and ulcer formation starts with a predisposed susceptibility for injury at baseline. A loss of protective sensation can substantially decrease the perception of touch, pain, deep pressure, temperature, and joint position. ${ }^{32}$ Once an open wound is present, the surrounding normal skin provides a base structure for the fragile granulation tissue. The surrounding skin in diabetes is less suited for this function, and trauma to the area is more likely to disrupt the developing wound matrix due to the dysregulated inflammation and proteolysis that occurs in diabetic wounds.

Although the exact mechanism for the inferior biomechanical properties of diabetic skin at baseline remains to be established, there are several potential etiologies. It has previously been reported in the literature that diabetic skin has a higher type III to I collagen ratio than nondiabetic skin. ${ }^{33}$ This relative increase in type III collagen might account for the decreased strength and stiffness of diabetic skin.

Although our results demonstrate some significant differences, as well as the trends toward significance with respect to MMP-2, MMP-9, and TIMP-1 levels, these have not fully clarified the pathophysiology leading to decreased tensile strength. Clinical data suggest that diabetic wounds that have a greater propensity for healing have lower MMP activity, higher levels of TIMP-1 and greater ratios of MMP-9 to TIMP-1. ${ }^{34}$ Our results do not reflect a similar ratio, but no other studies have investigated production of these proteins at baseline.

Perhaps most elucidating in our study was discovering that the level of mature collagen I and collagen III were both lower in the diabetic mouse. This is surprising when taken into context of the RT-PCR findings, demonstrating greater expression of collagen synthesis genes in the diabetic mouse. This leads us to believe there is a post-transcriptional modification that leads to decreased collagen synthesis and also subsequently hydroxylation and cross linking in the diabetic mouse. Our RT-PCR data are consistent with overall protein levels in both groups of mice. Our data suggests that in fact the ECM of a phenotypically normal mouse or patient undergoes greater proteolysis at baseline secondary to greater MMP-9, TIMP-1, and MMP-9:TIMP-1 ratio. Although contrary to our initial hypothesis, our findings can be explained by overall less stress to skin at baseline requiring less collagen remodeling.

Post-transcriptional regulation is a well-described molecular phenomenon. ${ }^{35}$ Two potential mechanisms are ribonuclease digestion of mRNA, which is transcribed, and microRNA that are able to silence translation of certain mRNAs through binding of complementary $3^{\prime}$ untranslated region (UTR) sequences. For instance, the half-life of casein mRNA is lengthened when prolactin is present, thus improving milk production. ${ }^{36}$ Alternatively, studies have demonstrated that lack of microRNA production can permit uncontrolled cellular proliferation in the setting of leukemia ${ }^{37}$ and breast cancer. ${ }^{38}$ Recently, Roggli et al ${ }^{39}$ demonstrated in a mouse diabetic model that inflammatory cytokines elicit production of microRNA species that may be involved in the development of pancreatic $\beta$-cell failure, whereas the direct connection between increased production of microRNA targeting collagen gene expression and diabetes is still unclear. This pathophysiology can certainly explain our findings, but 
of course, more investigation is necessary of to further evaluate the role microRNA species play in the development of diabetes.

Having identified the inferior biomechanical properties of diabetic skin at baseline, there is the potential to develop new therapeutic strategies. If local treatments can be developed to improve both the strength and stiffness of diabetic skin, they would only need to be applied locally to high-risk areas. By increasing local strength and stiffness, skin that is exposed to repetitive minor trauma may become more resilient and less susceptible to injury. Improving the biomechanical properties of diabetic skin at baseline has the potential to lead to a reduction in the morbidity and economic costs related to diabetes.

\section{Acknowledgments}

We thank Haiying Li (Center for Fetal Research at the Children's Hospital of Philadelphia) for her contribution to performing the Western blots.

\section{References}

1. Gu K, Cowie CC, Harris MI: Mortality in adults with and without diabetes in a national cohort of the U.S. population, 1971-1993. Diabetes Care 1998, 21:1138-1145

2. Most RS, Sinnock P: The epidemiology of lower extremity amputations in diabetic individuals. Diabetes Care 1983, 6:87-91

3. Brem H Tomic-Canic M: Cellular and molecular basis of wound healing in diabetes. J Clin Invest 2007, 117:1219-1222

4. Falanga V: Wound healing and its impairment in the diabetic foot. Lancet 2005, 366:1736-1743

5. Nilsson J, Bengtsson E, Fredrikson GN: Bjorkbacka. Inflammation and immunity in diabetic vascular complications. Curr Opin Lipidol 2008, 19:519-524

6. Badillo AT, Redden RA, Zhang L, Doolin EJ, Liechty KW: Treatment of diabetic wounds with fetal murine mesenchymal stromal cells enhances wound closure. Cell Tissue Res 2007, 329:301-311

7. Lobmann R, Ambrosch A, Schultz G, Waldmann K, Schiweck S, Lehnert $\mathrm{H}$ : Expression of matrix-metalloproteinases and their inhibitors in the wounds of diabetic and nondiabetic patients. Diabetologia 2002, 45:1011-1016

8. Neely AN, Clendening CE, Gardner J, Greenhalgh DG: Gelatinase activities in wounds of healing-impaired mice versus wounds of nonhealing-impaired mice. J Burn Care Rehabil 2000, 21:395-402

9. Hennessey PJ, Ford EG, Black CT, Andrassy RJ: Wound collagenase activity correlates directly with collagen glycosylation in diabetic rats. J Pediatr Surg 1990, 25:75-78

10. Brownlee M: Advanced protein glycosylation in diabetes and aging Annu Rev Med 1995, 46:223-234

11. Aronson D: Cross-linking of glycated collagen in the pathogenesis of arterial and myocardial stiffening of aging and diabetes. J Hypertens 2003, 21:3-12

12. Goova MT, Li J, Kislinger T, Qu W, Lu Y, Bucciarelli LG, Nowygrod S, Wolf BM, Caliste X, Yan SF, Stern DM, Schmidt AM: Blockade of receptor for advanced glycation end-products restores effective wound healing in diabetic mice. Am J Pathol 2001, 159:513-525

13. Peppa M, Stavroulakis P, Raptis SA: Advanced glycoxidation products and impaired diabetic wound healing. Wound Repair Regen 2009, 17:461-472

14. Wetzler $\mathrm{C}$, Kampfer $\mathrm{H}$, Stallmeyer B, Pfeilschifter J, Frank S: Large and sustained induction of chemokines during impaired wound healing in diabetic mouse: prolonged persistence of neutrophils and macrophages during the late phase of repair. J Invest Dermatol 2000, 115:245-253

15. Andreassen TT, Oxlund $\mathrm{H}$ : The influence of experimental diabetes and insulin treatments on the biochemical properties of rat skin incisional wounds. Acta Chir Scand 1987, 153:405-409
16. Soslowsky LJ, Thomopoulos S, Tun S, Flanagan CL, Keefer CC Mastaw J, Carpenter JE: Neer Award 1999. Overuse activity injures the supraspinatus tendon in an animal model: a histologic and biomechanical study. J Shoulder Elbow Surg 2000, 9:79-84

17. Favata M. Scarless Healing in the Fetus: Implications and Strategies for Postnatal Tendon Repair (dissertation). [Philadelphia (PA)]: University of Pennsylvania

18. Christner PJ, Gentiletti J, Peters J, Ball ST, Yamauchi M, Atsawasuwan P, Beason DP, Soslowsky LJ, Birk DE: Collagen dysregulation in the dermis of the Sagg/+ mouse: a loose skin model. J Invest Dermatol 2006, 126:595-602

19. Derwin KA, Soslowsky LJ, Green WD, Elder SH: A new optical system for the determination of deformations and strains: calibration characteristics and experimental results. J Biomech 1994, 27:1277-1285

20. Schram K, Wong MM, Palanivel R, No EK, Dixon IM, Sweeney G: Increased expression and cell surface localization of MT1-MMP plays a role in stimulation of MMP-2 activity by leptin. J Mol Cell Cardiol 2008, 44:874-881

21. Miller EJ, Rhodes RK: Preparation and characterization of the different types of collagen. Methods Enzymology 1982, 82:33-64

22. Stadelmann WK, Digenis AG, Tobin GR: Physiology and healing dynamics of chronic cutaneous wounds. Am J Surg 1998, 176:26S-38S.

23. Birk DE, Mayne R: Localization of collagen types I. III and V during tendon development Changes in collagen types I and III are correlated with changes in fibril diameter Eur J Cell Biol 1997, 72 : 352-361

24. Reihsner R, Melling M, Pfeiler W, Menzel EJ: Alterations of biochemical and two-dimensional biomechanical properties of human skin in diabetes mellitus as compared to effects of in vitro non-enzymatic glycation. Clin Biomech 2000, 15:379-86

25. Nikkels-Tassoudji N, Henry F, Letawe C, Pierard-Franchimont C, Lefebvre P, Pierard GE: Mechanical properties of the diabetic waxy skin. Dermatology 1996, 192:19-22

26. Singh N, Armstrong DG, Lipsky BA: Preventing foot ulcers in patients with diabetes. JAMA 2005, 293:217-228

27. Yang C, Zhu P, Yan L, Chen L, Meng R, Lao G: Dynamic changes in matrix metalloproteinase 9 and tissue inhibitor of metalloproteinase 1 levels during wound healing in diabetic rats. J Am Podiatr Med Assoc 2009, 99:489-496

28. Krampert M, Kuenzle S, Thai SN, Lee N, Iruela-Arispe ML, Werner S: ADAMTS1 proteinase is up-regulated in wounded skin and regulates migration of fibroblasts and endothelial cells. A J Biol Chem 2005, 280:23844-23852

29. Liao H, Zakhaleva J, Chen W: Cells and tissue interactions with glycated collagen and their relevance to delayed diabetic wound healing. Biomaterials 2009, 30:1689-1696

30. Seibold JR, Uitto J, Dorwart BB, Prockop DJ: Collagen synthesis and collagenase activity in dermal fibroblasts from patients with diabetes and digital sclerosis. J Lab Clin Med 1985, 105:664-667

31. Rittié L, Berton A, Monboisse JC, Hornebeck W, Gillery P: Decreased contraction of glycated collagen lattices coincides with impaired matrix metalloproteinase production. Biochem Biophys Res Commun 1999, 264:488-492

32. Cavanagh PR, Lipsky BA, Bradbury AW, Botek G: Treatment for diabetic foot ulcers. Lancet 2005, 366:1725-35

33. Kern P, Moczar M, Robert L: Biosynthesis of skin collagens in normal and diabetic mice. Biochem J 1979, 182:337-345

34. Ladwig GP, Robson MC, Liu R, Kuhn MA, Muir DF, Schultz GS: Ratios of activated matrix metalloproteinase-9 to tissue inhibitor of matrix metalloproteinase- 1 in wound fluids are inversely correlated with healing of pressure ulcers. Wound Repair Regen 2002, 10: $26-37$

35. Mattick JS: RNA regulation: a new genetics? Nat Rev Genet 2004, 5:316-323

36. Forsyth IA, Wallis M: Growth hormone and prolactin-molecular and functional evolution. J Mammary Gland Biol Neoplasia 2002, 7:291-312

37. Zhang B, Pan X, Cobb GP: microRNAs as oncogenes and tumor suppressors. Dev Biol 2007, 302:1-12

38. Heneghan HM, Miller N, Lowery AJ, Sweeney KJ, Kerin MJ: MicroRNAs as novel biomarkers for breast cancer. J Oncol 2009, 2009:950201

39. Roggli E, Britan A, Gattesco S, Lin-Marq N, Abderrahmani A, Meda P, Regazzi R: Involvement of microRNAs in the cytotoxic effects exerted by proinflammatory cytokines on pancreatic beta-cells. Diabetes 2010, 59: 978-986 